\title{
Improved strong magnetic field performance of low angle grain boundaries of calcium and oxygen overdoped $\mathrm{YBa}_{2} \mathrm{Cu}_{3} \mathrm{O}_{x}$
}

\author{
George A. Daniels, ${ }^{\text {a) }}$ Alex Gurevich, and David C. Larbalestier \\ Applied Superconductivity Center and Department of Materials Science and Engineering, \\ University of Wisconsin, Madison, Wisconsin 53706
}

(Received 28 July 2000; accepted for publication 6 September 2000)

\begin{abstract}
Critical currents and extended voltage-current characteristics of [001] tilt, low angle boundaries in thin film bicrystals of pure $\mathrm{YBa}_{2} \mathrm{Cu}_{3} \mathrm{O}_{x}$ and $\mathrm{Y}_{0.7} \mathrm{Ca}_{0.3} \mathrm{Ba}_{2} \mathrm{Cu}_{3} \mathrm{O}_{x}$ in strong magnetic fields $(H<10 \mathrm{~T})$ have been measured. For $5^{\circ}[001]$ tilt boundaries, there are clear benefits to $\mathrm{Ca}$ additions, particularly when the sample is oxygen treated to further overdope the sample after growth. Ca-doping decreases excess grain boundary resistance and diminishes the gap between inter- and intragrain current density over a wide field and temperature range. In spite of the lower $T_{c}$ of the $0.3 \mathrm{Ca}$-doped samples, Ca doping increases the intergrain critical current density at $44 \mathrm{~K}$ as high as $30 \%$ over $0-3$ $\mathrm{T}$ and threefold at 5 T. (C) 2000 American Institute of Physics. [S0003-6951(00)03644-5]
\end{abstract}

Mechanisms of current transport through grain boundaries (GB) in high-temperature superconductors and the critical misorientation angle $\theta_{c}$ at which a GB obstructs supercurrent flow are of great interest. ${ }^{1-3}$ These issues are particularly important for biaxially textured coated conductors, ${ }^{4-6}$ where recent magneto-optical studies suggest that the intergranular critical current density $J_{b}$ falls below the intragrain current density $J_{c}$ for $\theta \geqslant 4^{\circ}{ }^{7}$ A low angle $\left(\theta<5^{\circ}-10^{\circ}\right)[001]$ tilt GB can be modeled as an array of edge dislocations with insulating cores of the order of the Burgers' vector $(0.4 \mathrm{~nm})$ in diameter with relatively undisturbed channels between the cores. The quality of the channels and the charge imbalance on the GB play a large role in determining $J_{b}{ }^{8,9}$ Screening of the excess GB charge leads to a depression of the superconducting order parameter in the channels and over a region of 1-2 $\mathrm{nm}$ near the GB. A model ${ }^{8}$ which incorporates these characteristics, explains the quasiexponential decline of $J_{b}(\theta)$ observed for large $\theta$ in YBCO bicrystals, ${ }^{1-3}$ indicating that $J_{b}(\theta)$ can be improved by increasing the GB carrier density (for example, by overdoping the sample). However, since $\mathrm{YBa}_{2} \mathrm{Cu}_{3} \mathrm{O}_{x}$ can only be slightly overdoped from the optimal composition of $x=6.94$ up to $x=7$, their GBs are generally thought to be hole deficient. ${ }^{10,11}$ Recently, Schmehl et al. ${ }^{12}$ have made Cadoped $\mathrm{YBa}_{2} \mathrm{Cu}_{3} \mathrm{O}_{x}$ bicrystals where greater overdoping is possible, ${ }^{13}$ finding that the GB resistance could be strongly decreased and $J_{b}(0 \mathrm{~T}, 4.2 \mathrm{~K})$ of some $24^{\circ}$ [001] tilt GBs increased by as much as eight times. More recent work with $\mathrm{YBa}_{2} \mathrm{Cu}_{3} \mathrm{O}_{x} / \mathrm{Y}_{0.7} \mathrm{Ca}_{0.3} \mathrm{Ba}_{2} \mathrm{Cu}_{3} \mathrm{O}_{x}$ multilayers showed that $J_{b}(0 \mathrm{~T}, 77 \mathrm{~K})$ could be raised by up to 3.5 times. ${ }^{14}$ However, these dramatic increases in $J_{b}(\theta)$ were observed in zero magnetic field on high angle GBs which remained weakly coupled even after $\mathrm{Ca}$ overdoping. In the present work we report on the benefits of overdoping with $\mathrm{Ca}$ and $\mathrm{O}$ for low angle GBs. We measured the extended voltage-current $(V-I) \quad$ characteristics of $5^{\circ}$ and $7^{\circ}$ [001] tilt $\mathrm{Y}_{0.7} \mathrm{Ca}_{0.3} \mathrm{Ba}_{2} \mathrm{Cu}_{3} \mathrm{O}_{x}$ bicrystals in magnetic fields up to $10 \mathrm{~T}$. We found considerable increase of $J_{b}(\theta)$ due to the Ca overdoping.

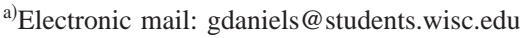

Thin films were grown on [001]-oriented $\mathrm{SrTiO}_{3}$ bicrystals by pulsed laser deposition under identical conditions using both pure $\mathrm{YBa}_{2} \mathrm{Cu}_{3} \mathrm{O}_{x}$ and $\mathrm{Y}_{0.7} \mathrm{Ca}_{0.3} \mathrm{Ba}_{2} \mathrm{Cu}_{3} \mathrm{O}_{x}$ targets. ${ }^{13}$ Our films were grown at 210 mTorr oxygen pressure and a substrate temperature of $810^{\circ} \mathrm{C}$, followed by a postgrowth annealing in oxygen at 830 Torr and $520^{\circ} \mathrm{C}$ for $30 \mathrm{~min}$ before final cooldown. Most data was obtained on two $5^{\circ}$ [001] tilt bicrystals. Cross-sectional scanning electron microscopy showed the film thickness of the pure and Ca-doped films to be 260 and $228 \mathrm{~nm}$, respectively. Bridges $25 \mu \mathrm{m}$ wide were patterned using lithography and argon ion beam etching techniques. Each bridge contained voltage taps for voltage measurement of the intergrain $(26 \mu \mathrm{m}$ long) and adjacent intragrain $(240 \mu \mathrm{m}$ long) portions of the bridge. $V-I$ characteristics were measured over 4-5 decades of $V$ using Keithley 2001 nanovoltmeters (sensitivity better than $5 \mathrm{nV}$ ). Both $J_{c}$ and $J_{b}$ were defined using the standard electric field criteria of $1 \mu \mathrm{V} / \mathrm{cm}$. The $\mathrm{Y}_{0.7} \mathrm{Ca}_{0.3} \mathrm{Ba}_{2} \mathrm{Cu}_{3} \mathrm{O}_{x}$ film was measured in its as-grown condition before subsequent postannealing (in pure oxygen for $30 \mathrm{~min}$ at $420^{\circ} \mathrm{C}$ ) and retesting in the oxygen overdoped state. The pure $\mathrm{YBCO}$ films had narrow resistive transitions $(\Delta T<0.4 \mathrm{~K})$. As-grown Cadoped films had broader transitions $(\Delta T<4 \mathrm{~K})$, sharpening $(\Delta T<1.2 \mathrm{~K})$ upon postoxygen annealing. The zeroresistance $T_{c}$ values were 91 and $78 \mathrm{~K}$ for the pure and Ca-doped films in the as-grown condition, but the $T_{c}$ of the Ca-doped film fell to $71 \mathrm{~K}$ after postoxygenation.

Figure 1 compares the $E-J$ curves for the intergrain and the intragrain links taken at a constant reduced temperature, $t=T / T_{c}=0.85(77 \mathrm{~K}$ for the pure and $66 \mathrm{~K}$ for the as-grown Ca-doped $5^{\circ}$ [001] tilt bicrystal). Both films exhibit singlecrystal-like intragrain $E-J$ characteristics, the curvature of $E(J)$ changing smoothly from negative to positive with increasing $H$. We used the change of sign of the curvature of $E(J)$ to define the irreversibility field, $H^{*}=5 \mathrm{~T}$ for pure YBCO and $3 \mathrm{~T}$ for Ca-doped YBCO at $t=0.85$. In spite of its lower $H^{*}$, the Ca-doped film had higher absolute $J_{c}$ values at lower fields. The intergrain $E-J$ characteristics of the Cadoped film are single-crystal-like, unlike those of the pure YBCO film, where the low field (1-3 T) intergrain $E-J$ curves show an initially steep rise followed by a pronounced kink at higher $E$. As shown later, this behavior is due to flux 

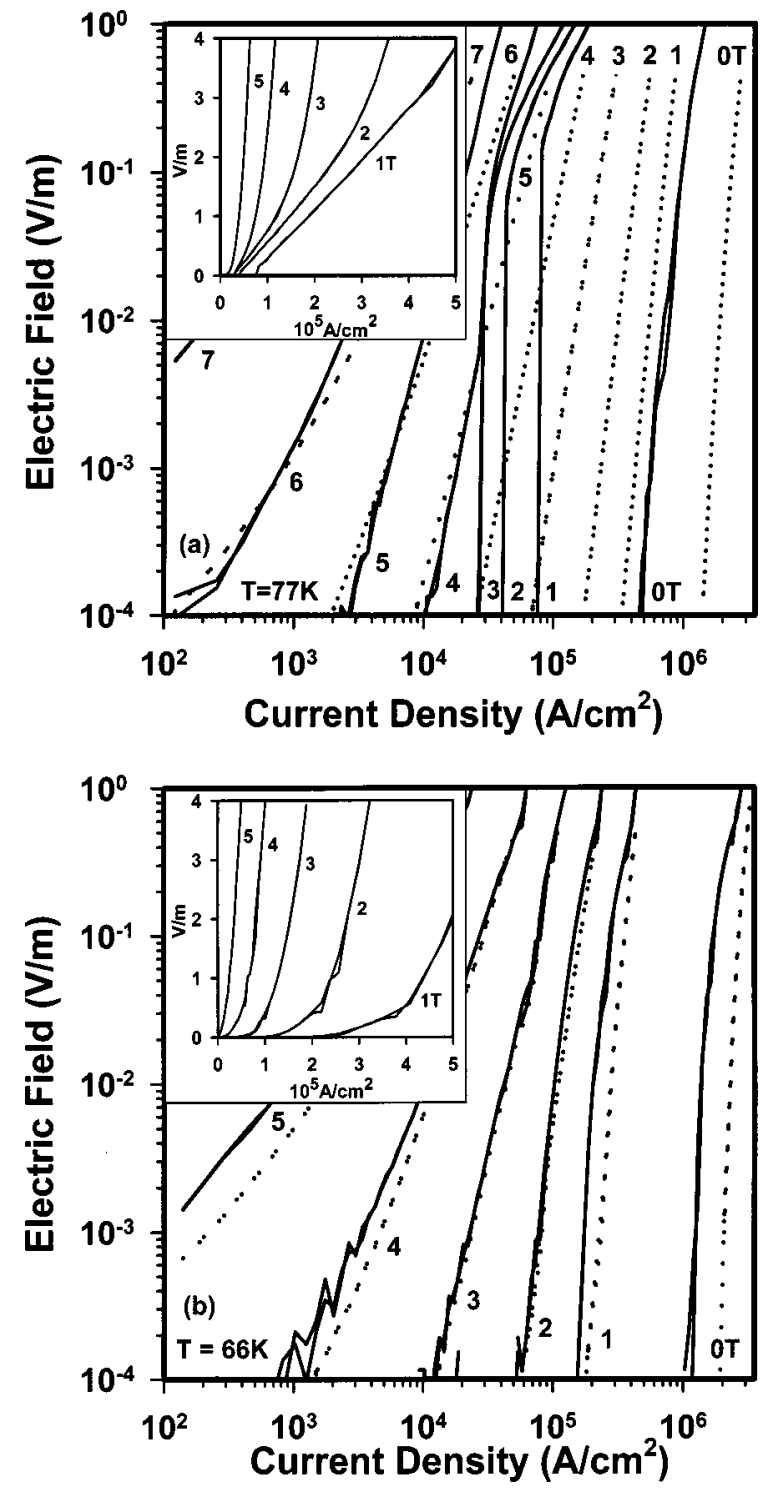

FIG. 1. Intragrain (dotted) and intergrain (solid) $E-J$ characteristics of the pure YBCO [1(a)] and Ca-doped [1(b)] $5^{\circ}$ [001] tilt bicrystal films taken at the same reduced temperature $t=T / T_{c}=0.85$, that is 77 and $66 \mathrm{~K}$, respectively. Magnetic fields (in tesla) are shown at the top and bottom of each trace. The insets show the intergrain $E-J$ curves on a linear scale.

flow along the GB. The pure YBCO film at $4 \mathrm{~T}$ demonstrates a marked change in the $E-J$ curve: the low-voltage part is intragrain-like, there then being a distinct change as the grain boundary dissipation takes over, changing again at the highest voltage to dissipation dominated by the intragrain components of the bridge. In spite of the lower irreversibility field of the Ca-doped grains, the Ca-doped sample has a higher intergrain current density than pure YBCO evaluated at the same $T / T_{c}$ of 0.85 .

Figure 2 compares $J_{b}$ and $J_{c}$ over a wide field and temperature space for pure and Ca-doped YBCO. For pure YBCO, there is a large difference between $J_{b}(H, T)$ and $J_{c}(H, T)$, while this difference is absent at higher temperatures for the oxygen-annealed, Ca-doped film. The as-grown Ca-doped film shows intermediate properties. Overall, $J_{b}(T)$ and $J_{c}(T)$ curves are always closer for the Ca-doped film than for the pure YBCO bicrystal, and oxygen-overdoping brings them even closer. Figure 3 compares the $J(H)$ curves at $44 \mathrm{~K}(t=0.48$,

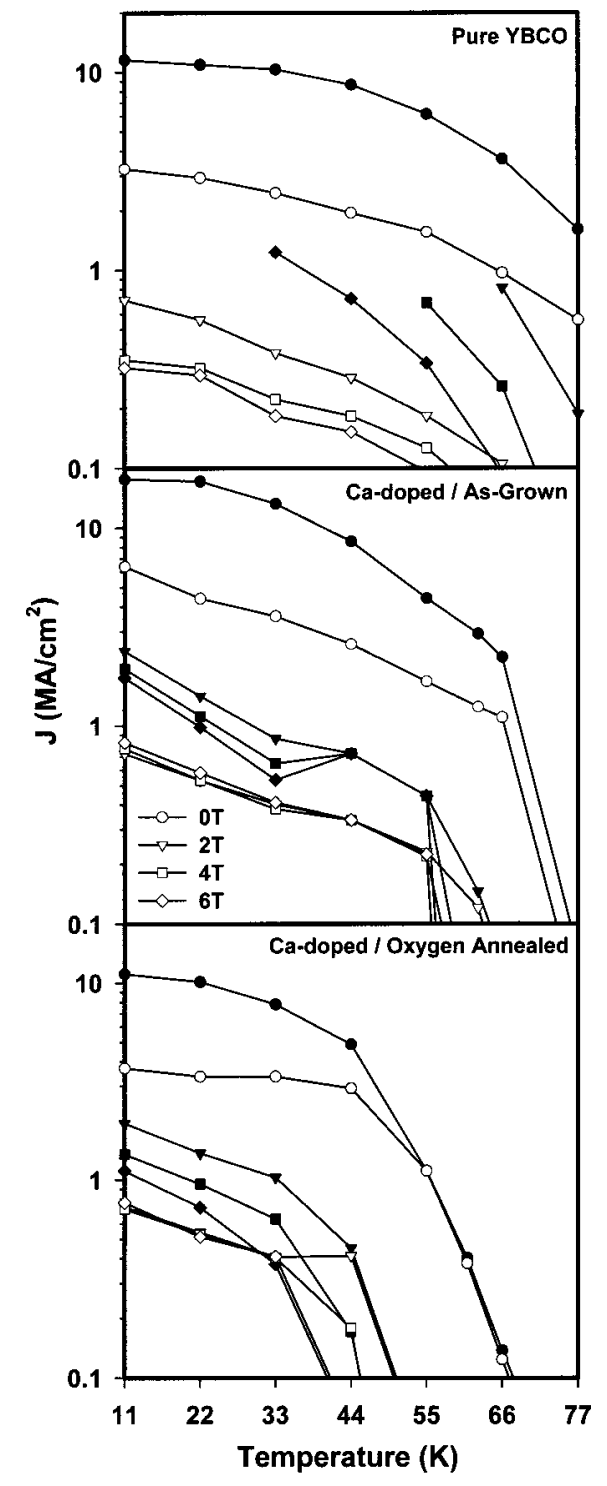

FIG. 2. Intergrain $J_{b}(T, H)$ (open symbols) and intragrain $J_{c}(T, H)$ (solid symbols) for different magnetic fields and doping states. The incomplete $J_{c}(T)$ curves for the pure YBCO result from the measurement configuration in which the intergrain voltage reaches its limit (due to Joule heating) before the intragrain dissipation has begun. This effect was not observed on the Ca-doped film due to smaller differences between $J_{b}(T)$ and $J_{c}(T)$.

0.56 , and 0.62 , respectively, for pure $\mathrm{YBCO}$, as-grown $\mathrm{Ca}-$ doped YBCO, and O-overdoped Ca-doped YBCO). The gap between $J_{b}(H)$ and $J_{c}(H)$ is very large for the pure YBCO, smaller for the as-grown Ca-doped sample, and is nearly erased for the oxygen-overdoped sample at $H>2$ T. Most significantly, oxygen overdoping the $\mathrm{Ca}$ sample notably increases $J_{b}(H)$ in fields up to $2.5 \mathrm{~T}$.

To clarify the pronounced kinks in the $E-J$ curves of the pure YBCO bicrystal, we plotted them in linear coordinates (Fig. 1 inset). The data shown in the inset in Fig. 1(a) are well described by a linear dependence $V=\left(I-I_{b}\right) R$, where $R(H)$ is an excess GB resistance, and $I_{b}$ the intergranular critical current. Similar behavior has also been observed on other YBCO bicrystals. ${ }^{15,16}$ The kinks of the log$\log V(I)$ plot in Fig. 1(a) thus result from the switching of the GB into a resistive state above $I_{b}$, while the displaced ohmic dependence $V=\left(I-I_{b}\right) R$ at $I \approx I_{b}$ indicates flux flow of vortices in a channel along the GB. For $H<4 \mathrm{~T}$, the $V-I$ curves are linear near the transition, $I \approx I_{b}(H)$, curving upto AIP license or copyright, see http://apl.aip.org/api/copyright.jsp 


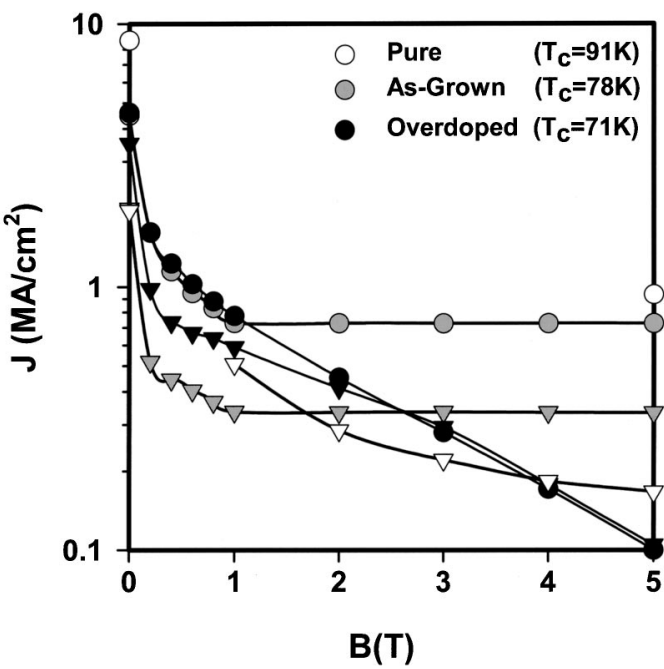

FIG. 3. Comparison of magnetic field dependencies of $J_{b}(H)(\boldsymbol{\nabla})$ and $J_{c}(H)(\bullet)$ for pure and Ca-substituted films in two different doping states at $44 \mathrm{~K}(t=0.47,0.56$, and 0.62 for pure, as grown and O-annealed Ca-doped films, respectively). Only two data points were available for the pure YBCO intragrain $J_{c}$ due to concerns about overheating the intergrain link.

ward at higher $I$, then becoming linear again at the highest accessible currents. The slopes $R(H)$ of the linear portion of $E(J)$ at $I \approx I_{b}(H)$ remain approximately constant from 1-3 $\mathrm{T}$, whereas the slopes $d E / d J$ for higher currents increase with $H$. These features can be described by a model of a flux flow channel ${ }^{15-17}$ which appears at $I=I_{b}$ and gradually expands with increasing $I$, occupying the whole sample as $I$ reaches $I_{c}$ of the grains. The expansion of the flux flow channel with increasing $I$, manifests itself as an upturn on the $\log -\log V-I$ curves above the kinks in Fig. 1(a). This picture is consistent with the nearly linear increase of the slope $d V / d I$ in the high $J$ region in the inset of Fig. 1(a), as expected from the Bardeen-Stephen model.

The flux flow channel can either comprise a single vortex row on the GB, or additional vortex rows in the grains. In the latter case we should expect a pronounced field dependence of the GB resistance, $R \cong H w(H, J) \rho_{n} / A H_{c 2}$, where $\rho_{n}$ is the normal state resistivity, $H_{c 2}$ is the upper critical field, $A$ is the cross-sectional area, and $w(H, J)$ is the channel width, which exceeds the intervortex spacing $a$ $=\left(\phi_{0} / H\right)^{1 / 2}$. However, at $I \approx I_{b}$, the effective GB resistance in Fig. 1(a) remains approximately constant as $H$ increases from 1 to $3 \mathrm{~T}$. The fact that $R(H)$ is nearly independent of $H$, as compared to the three-fold increase of the slope $d V / d I$ at higher $I$, indicates that there is only a single row of overlapped Josephson vortex cores moving along the GB. In this case $R$ is just the excess normal resistance of the GB For a low-angle GB, the length of the Josephson core equals $l$ $=\lambda_{J}^{2} / \lambda$ for $J_{b}>J_{d} / \kappa$, and $l=\lambda_{J}$ for $J_{b}<J_{d} / \kappa$, ${ }^{16}$ where $\lambda$ is the London penetration depth, $\lambda_{J}$ is the Josephson penetration depth, $J_{d}$ is the depairing current density, and $\kappa \cong 10^{2}$ is the Ginzburg-Landau parameter. Using these expressions for $l$, we can show that the condition that the vortex cores overlap, $l<\left(\phi_{0} / H\right)^{1 / 2}$, is indeed satisfied, even for the lowest field (1 T) in Fig. 1.

By contrast, the $V-I$ curves for the Ca-doped films [Fig. 1(b)] do not exhibit the pronounced kinks and steep slopes seen in $V(I)$ of pure films at $H<4 \mathrm{~T}$. This indicates that $\mathrm{Ca}$ doping strongly decreases the excess GB resistance $R$, so that the vortex slippage along the GB at $I>I_{b}$ does not manifest itself in the $V-I$ curves of the doped bicrystals. The conclusion is consistent with the significant decrease of $R$ observed on a Ca-doped $24^{\circ}$ [001] high-angle GBs. ${ }^{12}$ This reduction of $R$ results from the increase of the local carrier density at the hole-deficient GB with Ca doping. ${ }^{13}$ The overdoping thus partly compensates the suppression of the order parameter on the GB, which results from a progressive shift of the chemical potential $\mu(\theta)$ at the GB toward the antiferromagnetic insulating state as $\theta$ increases. ${ }^{8}$ Note that since $\mu(\theta)$ increases with $\theta$, the optimum Ca content may be different for GBs with different $\theta$.

In conclusion, the data of Figs. 1-3, taken over a wide range of electric field and current density, magnetic field and temperature all support the fact that $\mathrm{Ca}$ additions strongly benefit the intergranular current density of low angle GBs. More limited characterization of the other $5^{\circ}$ and $7^{\circ}[001]$ tilt GBs also qualitatively confirm this result. The present results support the models, ${ }^{8,9}$ and show benefits of $\mathrm{Ca}$ and $\mathrm{O}$ overdoping for low angle grain boundaries.

The authors are grateful to S. Liao and D. M. Feldmann for their aid in the film growth, to A. Squitieri and W. Starch for experimental assistance, and to S. E. Babcock and M. Rzchowski for discussions. The work has been supported by the NSF MRSEC (DMR No. 9214707). D. C. L. is grateful to The Department of Condensed Matter Physics at the University of Geneva for an invited professorship during the preparation of this manuscript.

${ }^{1}$ D. Dimos, P. Chaudhari, and J. Mannhart, Phys. Rev. B 41, 4038 (1990).

${ }^{2}$ N. F. Heinig, R. D. Redwing, J. E. Nordman, and D. C. Larbalestier, Phys. Rev. B 60, 1409 (1999)

${ }^{3}$ K. E. Gray, M. B. Field, and D. J. Miller, Phys. Rev. B 58, 9543 (1998).

${ }^{4}$ X. D. Wu, S. R. Foltyn, P. N. Arendt, W. R. Blumenthal, I. H. Campbell,

J. D. Cotton, J. Y. Coulter, W. L. Hults, M. P. Maley, H. F. Safar, and J. L. Smith, Appl. Phys. Lett. 67, 2397 (1995).

${ }^{5}$ A. Goyal, D. P. Norton, J. D. Budai, M. Paranthaman, E. D. Specht, D. M. Kroeger, D. K. Christen, Q. He, B. Saffian, F. A. List, D. F. Lee, P. M. Martin, C. E. Klabunde, E. Hartfield, and V. K. Sikka, Appl. Phys. Lett. 69, 1795 (1996).

${ }^{6}$ D. K. Finnemore, K. E. Gray, M. P. Maley, D. O. Welch, D. K. Christen, and D. M. Kroeger, Physica C 320, 1 (1999).

${ }^{7}$ D. M. Feldmann, J. L. Reeves, A. A. Polyanskii, G. Kozlowski, R. R. Biggers, R. M. Nekkanti, I. Maartense, M. Tomsic, P. Barnes, C. E. Oberly, T. L. Peterson, S. E. Babcock, and D. C. Larbalestier, Appl. Phys. Lett. (accepted for publication).

${ }^{8}$ A. Gurevich and E. A. Pashitskii, Phys. Rev. B 57, 13878 (1998).

${ }^{9}$ J. Mannhart and H. Hilgenkamp, Appl. Phys. Lett. 73, 265 (1998).

${ }^{10}$ S. E. Babcock and J. Vargas, Annu. Rev. Mater. Sci. 25, 193 (1995).

${ }^{11}$ N. D. Browning, J. P. Buban, P. D. Nellist, D. P. Norton, M. F. Chisholm, and S. J. Pennycook, Physica C 294, 183 (1998).

${ }^{12}$ A. Schmehl, B. Goetz, R. R. Schulz, C. W. Schneider, H. Bielefeldt, H. Hilgenkamp, and J. Mannhart, Europhys. Lett. 47, 110 (1999).

${ }^{13}$ J. T. Kucera and J. C. Bravman, Phys. Rev. B 51, 8582 (1995).

${ }^{14}$ G. Hammerl, A. Schmehl, R. R. Schulz, B. Goetz, H. Bielefeldt, C. W. Schneider, H. Hilgenkamp, and J. Mannhart, Nature (London) 407, 162 (2000)

${ }^{15}$ A. Díaz, L. Mechin, P. Berghuis, and J. E. Evetts, Phys. Rev. B 58, R2960 (1998).

${ }^{16}$ D. T. Verebelyi, D. K. Christen, R. Feenstra, C. Cantoni, A. Goyal, D. F. Lee, and M. Paranthaman, Appl. Phys. Lett. 76, 1755 (2000).

${ }^{17}$ A. Gurevich, Phys. Rev. B 46, R3187 (1992); 48, 12857 (1993); A. Gurevich and L. D. Cooley, ibid. 50, 13563 (1994). 